\title{
Three new families with recurrent male miscarriages and hypercoiled umbilical cord
}

Jodi D. Hoffman ${ }^{1}$, Linda Kleeman ${ }^{2}$, Kelley Kennelly ${ }^{3}$, Engela Honey ${ }^{4}$, William Reardon ${ }^{5}$

${ }^{1}$ Division of Genetics and ${ }^{2}$ Division of Maternal Fetal Medicine, Tufts Medical Center, Boston, MA, USA, ${ }^{3}$ St. Joseph Mercy Oakland, Pontiac, MI, USA, ${ }^{4}$ Department of Genetics, University of Pretoria, South Africa, ${ }^{5}$ National Maternity Hospital, Dublin 2, Ireland

Correspondence and reprint requests: Jodi D. Hoffman, MD, Division of Genetics, Tufts Medical Center, 800 Washington St., Box 340, Boston MA 02111, USA, jhoffman@tuftsmedicalcenter.org

Key Words: miscarriage, hypercoiling, umbilical coiling index

\section{Introduction}

In 2014, Beggan et al. described a family with two consecutive male pregnancy losses noted to have hypercoiling of the umbilical cord, postulating that there might be an unrecognized X-linked association which could present in this manner. Hypercoiling of the umbilical cord has been shown to be associated with fetal growth restriction and fetal heart rate decelerations (de Laat et al., 2006; Predanic M et al., 2005). Adverse perinatal outcomes are well established in cord hypercoiling, but are reported in both male and female babies. However, recurrent hypercoiling within a family had not been observed before this recent report.

Since Beggan's report, three further families have been identified in response to this publication and the original family has had another male loss. Hypercoiling of the cord, defined as umbilical cord index (complete coils divided by length in $\mathrm{cm}$ ) greater than the $90^{\text {th }}$ centile $(0.3 \mathrm{coils} / \mathrm{cm}$ in the mid-trimester per de Laat et al., 2007) was found in all cords examined in cases of male losses.

The association of abnormal umbilical cord coiling and adverse pregnancy outcome was first described by Strong in 1994. Both hypocoiling and hypercoiling have been associated with adverse perinatal 
outcomes (Strong, 1994). The mechanism of umbilical coiling remains unclear, but has been

hypothesized to result from fetal movement and uneven umbilical vessel growth rates (Finberg, 1992;

Chaurasia et al., 1979). It appears that umbilical coiling is protective against kinking of the blood vessels in utero and during parturition, but hypercoiling may result in compression and potentially thrombosis. Although the largest prospective study to date on the effect of umbilical cord coiling in an unselected patient population of 1,028 patients did not find an increased risk of adverse perinatal outcomes (Jessop et al., 2014), the role of abnormal coiling in a selected population with complicated pregnancies may be relevant (de Laat et al., 2007).

\section{Case Report - Family 1}

A non-consanguineous couple with three healthy daughters had three miscarriages of male fetuses, each noted to have hypercoiling of the umbilical cord. A detailed family history revealed no signs of the more common X-linked lethal conditions such as incontinentia pigmenti, chondrodysplasia punctata, Aicardi syndrome, oro-facial-digital I or any medical issues suggesting X-linked inheritance. Normal karyotypes were reported for both parents.

The first live child was a girl born at 41 weeks, later noted to have mild speech delay and light coloring with no known cause.

The first miscarriage occurred at a clinical gestational age (GA) of 18 weeks. The pregnancy had been otherwise uncomplicated and was naturally conceived. Pathologic evaluation after delivery revealed a macerated male fetus, appropriate for 14 weeks GA. No congenital malformations were noted. First trimester screening for aneuploidy was normal. The placenta had a hypercoiled cord and was marginally inserted. Laboratory testing at that time showed normal testing for thrombophilias (including APLAS), thyroid disease, lupus and maternal diabetes. 
After a chemical pregnancy, the next pregnancy resulted in the birth of dichorionic, diamniotic female twins following an IVF pregnancy due to new, unexplained intermittent infertility. Twin A is well and twin B has IUGR with speech and motor delays, narrowed aorta, and light iris pigmentation, with no genetic cause suspected.

The second miscarriage occurred at a clinical gestational age (GA) of 20 weeks after a spontaneous pregnancy. At delivery, this male fetus' length measured appropriate for 18-20 weeks GA while the weight was low for gestational age. The fetus had undergone autolysis. No congenital malformations were noted. The placenta had a hypercoiled cord, extreme narrowing at insertion of the cord, intervillous fibrin deposition, and notation of normal acylcarnitine studies. No karyotype was performed, due to lack of cell growth. (Fig. 1).

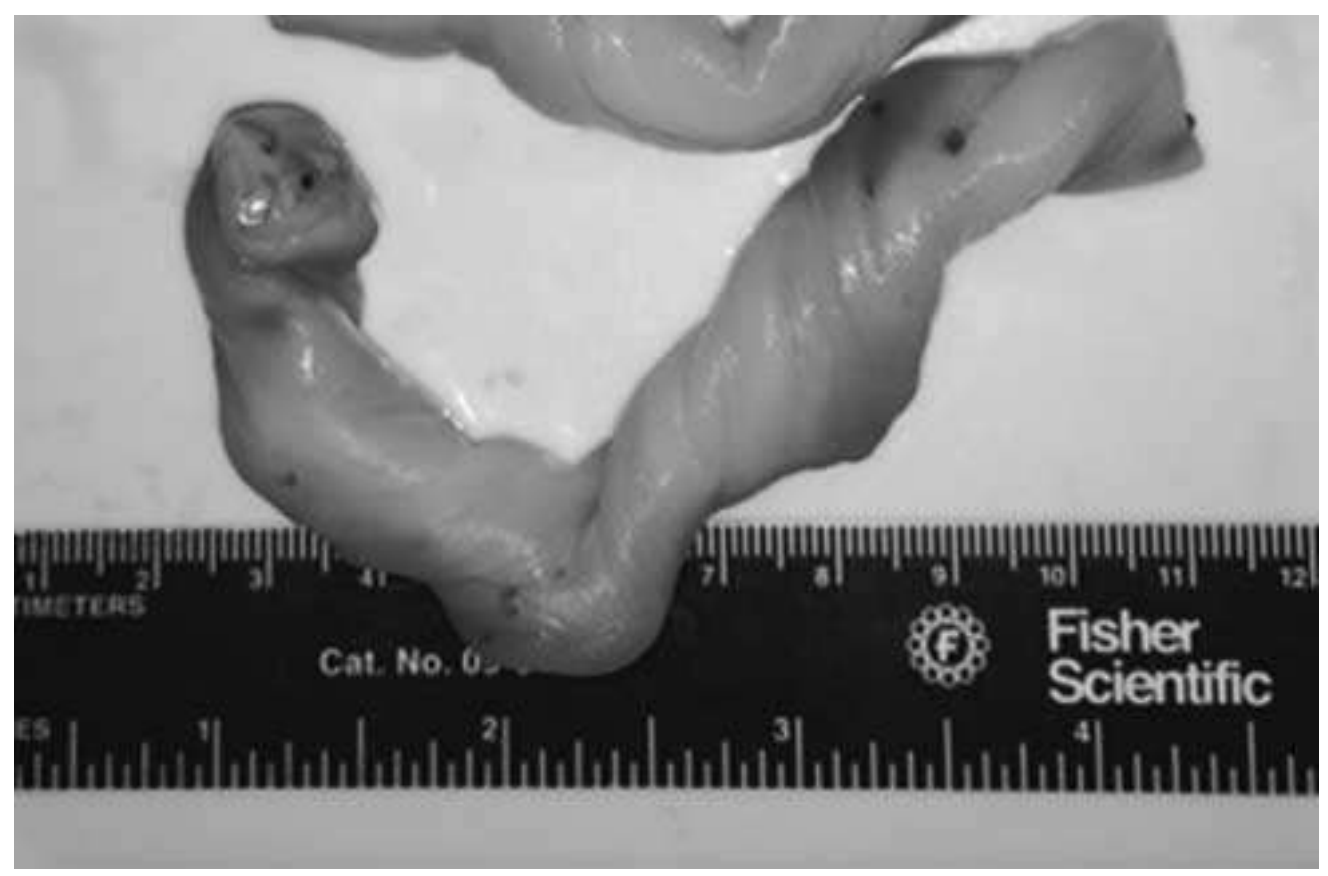

Figure 1: Normal cord showing typical degree of coiling

The third male fetus miscarriage occurred at 20 weeks clinical gestation and was conceived via frozen embryo transfer for infertility. The placenta had a hypercoiled cord (linked type) with UCl of 0.55 (6 coils 
in $11 \mathrm{~cm}$, Figure 2 ). The patient took $10 \mathrm{mg}$ citalopram daily (a selective serotonin uptake inhibitor) for depression during this pregnancy. Eight days prior to the loss, the fetus was noted to have discordant fetal biometry compared to last visit (10 days). Non-invasive prenatal screening showed no increased risk for trisomies. No karyotype was performed due to failure of cell growth.

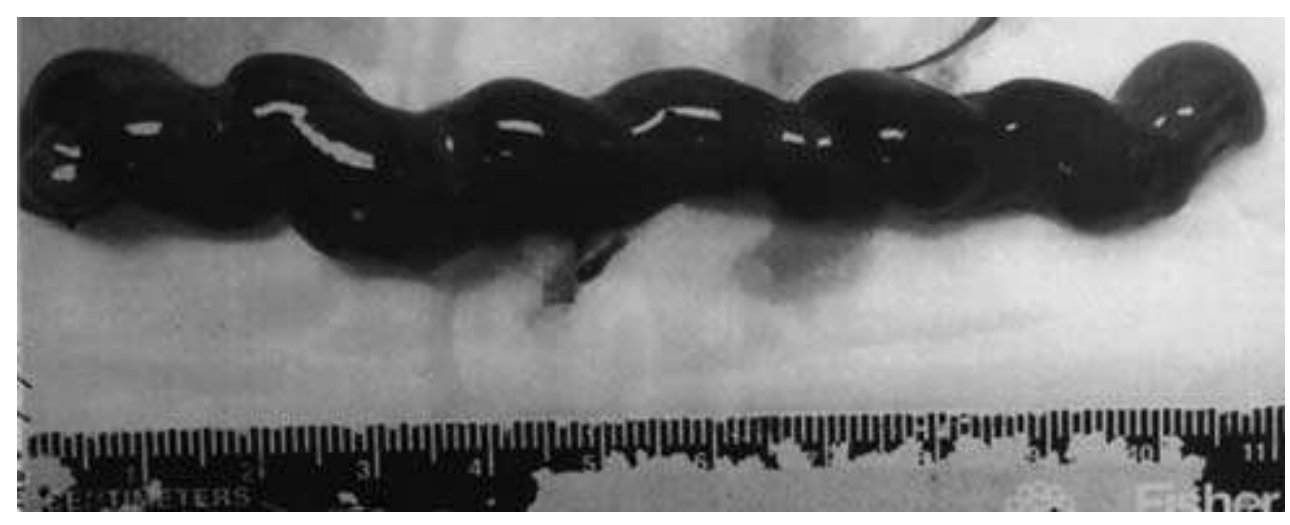

Figure 2: Cord of male fetus from family 1 showing hypercoiling (linked)

\section{Case Report -Family 2}

A non-consanguineous couple with four healthy daughters experienced two miscarriages of male fetuses; both were noted to have hypercoiling of the umbilical cord. Maternal age was 33 and 34 years, respectively, at the time of each miscarriage. A detailed four-generation family history did not reveal any symptoms that appeared to follow an X-linked pattern of inheritance or any evidence of the more common X-linked lethal conditions.

The first four pregnancies resulted in the birth of live female infants at term, all delivered by Caesarean section. No medical issues were noted prenatally or at birth for any of the four girls and they are all reported to be healthy. 
The first male fetal miscarriage occurred at a clinical gestational age of 21 weeks. The pregnancy up to that point had been uncomplicated and had occurred naturally. Ultrasound at 19 weeks showed no abnormalities and normal growth. Ultrasound at 21 weeks indicated an absence of fetal heart sounds. No congenital malformations were noted at delivery. Pathologic evaluation of the placenta described a hypercoiled cord with $\mathrm{UCl}$ of 0.86 (19 coils in $22 \mathrm{~cm}$ ) and paramarginal insertion. Fetal tissue was collected for cytogenetic analysis but no karyotype was possible due to lack of cell growth. Maternal serum screening and nuchal translucency measurements for aneuploidy were normal. Laboratory testing at that time for thyroid disease, thrombophilia, and autoimmune disease was all normal.

The second male fetal miscarriage occurred at a clinical gestational age of 16 weeks. The pregnancy was conceived naturally and was uncomplicated up to that point. Ultrasound at 16 weeks indicated an absence of fetal heart sounds. Measurements of femur length were consistent with a fetus of 15 weeks gestational age. Pathologic evaluation after delivery described a macerated male fetus with bilateral clubbed feet. No additional congenital malformations were noted. The placenta had a hypercoiled cord with $\mathrm{UCl}$ of 1.18 (13 coils in $11 \mathrm{~cm})$ and marginal insertion. Cell free fetal DNA analysis was normal. No chromosome analysis was performed.

Parental karyotypes were both normal.

\section{Case Report-Family 3}

A 32 year old woman presented with two intra-uterine losses at 20 weeks gestation during her first and second pregnancy. There was no consanguinity and no family history of note.

The first miscarriage followed an uncomplicated pregnancy and resulted in a well formed male fetus. Karyotype was normal male. The cord was noted to be "twisted" but unfortunately no other detail available and no further investigations were performed. 
The woman presented a year thereafter, pregnant with a male fetus. The pregnancy was normal to date and first trimester screening showed a low risk for chromosomal abnormalities. She experienced sudden loss of fetal movement at 20 weeks gestation and an intra-uterine death was confirmed on ultrasound. A well-formed male fetus (500g) was delivered. Karyotyping was unsuccessful but aneuploidy PCR showed no trisomy 13, 18, 21 or aneuploidy of the sex chromosomes. Culture of the amniotic fluid showed no viral infections and histology and microscopy and culture of the placenta was normal. The cord was noted to be hypercoiled (segmented type) with thrombosis of blood along the entire length of the cord (Figure 3).

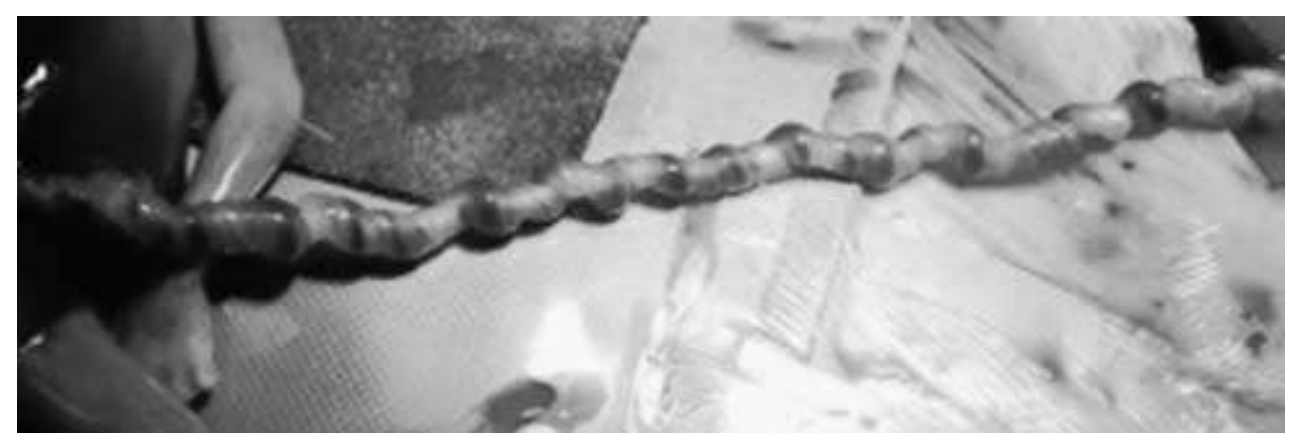

Figure 3: Cord of male fetus from family 3 showing hypercoiling (segmented)

\section{Additional information regarding previously reported case}

The mother of the index cases originally reported by Beggan et al., 2014 re-presented subsequent to the initial report. Following natural conception, pregnancy proceeded normally through the first trimester. Regular fetal ultrasound was consistent with normal growth and gave no cause for concern. At 16 weeks 4 days gestation intrauterine demise was noted on ultrasound and the male infant was delivered 2 days later, following medical induction. At delivery, the cord was tightly wrapped around the neck twice, with noticeable thinning of the umbilical cord around the abdominal insertion. 
Autopsy confirmed a normally formed, macerated, appropriately grown male infant. No fetal malformations were observed and there was no evidence of a congenital infection. The UCI was 1.25 (20 coils in $16 \mathrm{cms}$ ) consistent with hypercoiling. A karyotype failed but a $60 \mathrm{~Kb}$ array was normal. Maternal X-inactivation studies were normal, with no evidence of non-random inactivation.

\section{Discussion:}

As noted by Beggan et al. (2014), no publication had previously documented association of sex predilection with hypercoiled cord. We now report three additional families who have given birth to multiple healthy girls and had multiple male fetal losses, all with associated cord hypercoiling, as well as expanding the findings in the original reported family to include a third male loss. The cases we report show no other cause of death or congenital anomaly. We believe these emerging data support the original hypothesis that there may be an X-linked etiology to these cases. Taken as a whole, the 4 families described have had 7 female children all without clinical incident and 10 male early deaths in utero, ranging between 15 and 21 weeks, all associated with cord hypercoiling.

As family 1 had one daughter who had lighter coloring than expected for the family and one daughter with light colored irises, further assessment of this trait in the other families may be worthwhile, but is not available at this time.

Ernst et al. (2013) describe four patterns of hypercoiling seen in 318 cases of hypercoiled cords. Fifty-six percent of their stillbirth cases with hypercoiled cords had either the "linked" or "segmented" patterns of hypercoiling. These authors hypothesize that these hypercoiling subtypes may be more vulnerable to severe restriction of fetal blood flow. Unfortunately, we do not have images of several of the cases from our series available to further characterize the sub-type of hypercoiling. However, review of the images of two of our cases appear to show an example of the "linked" and "segmented" types. Further 
consideration of the subtypes of hypercoiling in future families with recurrent male pregnancy loss may suggest a mechanism for pregnancy losses in these cases.

Although the finding of a hypercoiled umbilical cord may not be etiologic to the fetal losses, the prenatal finding of a hypercoiled umbilical cord in families with a history of recurrent losses of male fetuses could point to identifying a previously undiagnosed cause of X-linked perinatal loss. We hope to move toward a genetic etiology via molecular studies of these families in an effort to further understand the basis of hypercoiling and male fetal losses.

Acknowledgements: We thank the pathologists on these cases- Masha Bilic, MD, Tufts Medical Center, Boston, MA; Faisal Quereshi, MD, Harper University Hospital, Detroit, MI; Paul Downey and Eoghan Mooney, National Maternity Hospital, Holles St, Dublin 2, Ireland

Conflict of interest: There are no conflicts of interest.

\section{References:}

Beggan C, Mooney EE, Downey P, Carroll S, Reardon W (2014).A case of recurrent familial male miscarriages with hypercoiled umbilical cord: A possible X-linked association? Clin Dys ; 23:26-28.

Chaurasia BD, Agarwal BM (1979). Helical structure of the human umbilical cord. Acta Anat;103:226-30.

de Laat MW, Franx A, Bots ML, Visser GH, Nikkels PG (2006). Umbilical coiling index in normal and complicated pregnancies, Obstetrics and Gynecology; 107(5): 1049-55.

de Laat MW, van Alderen ED, Franx A, Visser GH, Bots ML, Nikkels PG (2007). The umbilical coiling index in complicated pregnancy ; 130 (1):66-72.

Ernst LM, Minturn L, Huang MH, Curry E, Su EJ. Gross Patterns of Umbilical Cord Coiling: Correlations with Placental Histology and Stillbirth. Placenta 2013; (34): 583-8.

Finberg HJ (1992). Avoiding ambiguity in the sonographic determination of the direction of umbilical cord twist. J Ultrasound Med;11:185-9. 
Jessop FA, Lees CC, Pathak S, Hook CE, Sebire NJ (2014). Umbilical cord coiling: clinical outcomes in an unselected population and systematic review, Virchows Arch; 464:105-112.

Predanic M, Perni SC, Chasen ST, Baergen RN, Chervenak FA (2005). Ultrasound evaluation of abnormal umbilical cord coiling in second trimester of gestation in association with adverse pregnancy outcome. Am J Obstet Gynecol; 193:387-394.

Strong TH Jr, Jarles DL, Vega JS, Feldman DB (1994). The umbilical coiling index. Am J Obstet Gynecol;170:29-32 